\title{
LOW FREQUENCY ANTIPLANE SHEAR VIBRATIONS OF A THREE-LAYERED ELASTIC PLATE
}

\author{
Barış ERBAŞ \\ Eskişehir Technical University, Department of Mathematics,Yunus Emre Campus, Eskişehir, Turkey
}

\begin{abstract}
Antiplane shear vibrations of three-layered strongly vertically inhomogeneous laminates are considered. Polynomial long wave low frequency approximations of the exact dispersion relation are derived and further simplified for the two setups of material and geometric problem parameters corresponding, particularly, to laminated glass plates as well as photovoltaic panels. The associated 1D shortened equations of motion are also established using the conventional asymptotic procedure earlier developed for thin homogeneous structures.
\end{abstract}

Keywords: Asymptotic, Cut-off, Dispersion, Wave, Layered structure

\section{INTRODUCTION}

The manufacturing of multi-layered structures, particularly plates and shells composed of three layers, also known as sandwich plates, with geometrical symmetry and high contrast in material properties has long been in demand. An immediate example of such application is the manufacturing of windscreens in automotive industry as well as glazing of structures in civil engineering. The choice of lightweight materials coated with elements of strong flexural rigidity is one of the necessities required by modern aerospace, automotive, and civil engineering industries, e.g. see [1]. The high contrast of layer thicknesses, stiffness or densities is common in many industries, for example photovoltaic panels [2, 3], meta-materials [4]. Most of the structures like the ones just mentioned above are composed of very strong outer coatings with much softer inner layers. There are, however, structures composed of soft outer coatings with stiff inner layer such as precipitator plates found in gas filters to reduce air pollution [5]. There are numerous publications investigating the mechanics of multilayered structures involving sandwich plates and beams with a particular emphasis on high contrast problems, see $[6,7$, 8]. To the best of author's knowledge, the number of publications investigating strongly inhomogeneous structures are scarce. We mention here only the asymptotic investigations on the subject carried out in $[2,9,10,11,12]$.

This paper extends the results obtained in [13] to two more different configurations, namely, investigates the strongly inhomogeneous three-layered plate with thick stiff outer layers with soft inner layer and thin soft outer layers with stiff thick inner layer. The paper is organized as follows: in Section 2 the problem statement is formulated and the geometry of the problem investigated is presented. The dispersion relation is obtained for both cases. In Section 3, the equations derived in the previous section are nondimensionalised and the dispersion relation is presented as a Taylor series in the dimensionless variables. Section 4 considers the first configuration and reduces the dispersion relation to a more convenient form. Later, taking into account the orders of the problem parameters, a shortened dispersion relation is obtained. Section 5 repeats the analysis for the second configuration. Again, a shortened polynomial dispersion is presented. In both sections, the obtained results are compared with the exact dispersion relation given in Section 2. In Section 6, the asymptotic forms of the equations of motions presented in Section 2 are derived. It is demonstrated that the dispersion

*Corresponding Author: berbas@eskisehir.edu.tr

Received: 16.09.2018 Accepted: 16.11.2018 
relations obtained from the approximate formulas of equations of motion coincide with the shortened dispersion relations derived in Sections 4 and 5. Finally, the results obtained and possible extensions are discussed in the conclusions.

\section{STATEMENT OF THE PROBLEM}

Let us consider the anti-plane shear of three-layered elastic plate with inner layer of thickness $2 h_{1}$ and outer layers of thickness $h_{2}$ represented in Figure 1. Both inner and outer layers are assumed isotropic and the plate is symmetric with respect to its midplane.

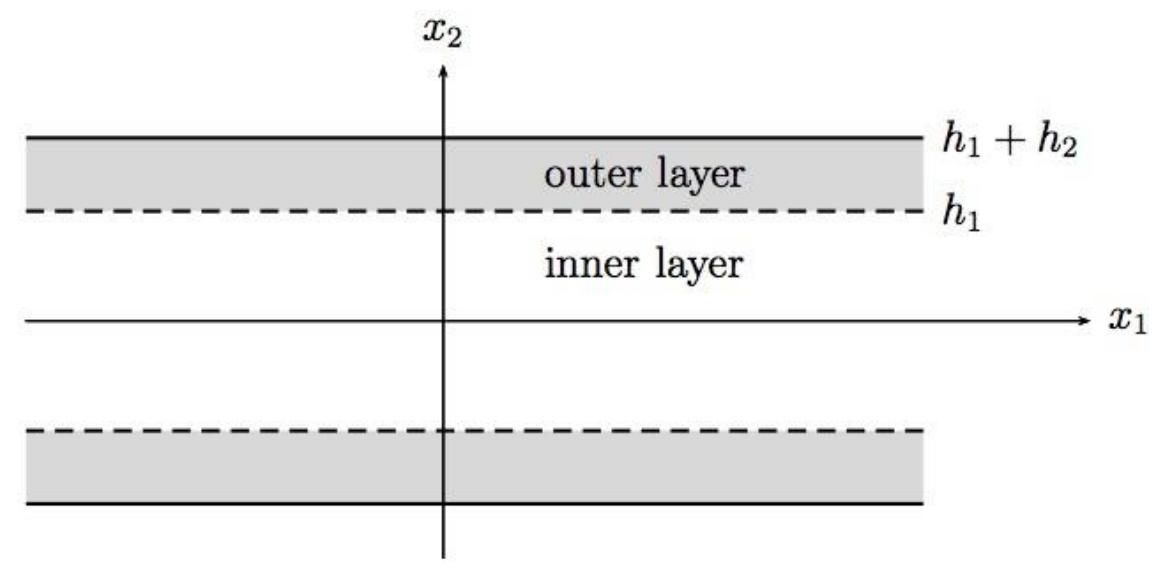

Figure 1. The geometry of the three-layered elastic layer.

The equations of motion, in terms of the cartesian coordinates $x_{n}, n=1,2,3$ can be written as (see, [14])

$$
\frac{\partial \sigma_{13}^{(i)}}{\partial x_{1}}+\frac{\partial \sigma_{23}^{(i)}}{\partial x_{2}}-\rho_{i} \frac{\partial^{2} u^{(i)}}{\partial t^{2}}=0, \quad i=1,2,
$$

where $t$ denotes time and $\sigma_{j 3}^{(i)}$ are the shear stresses related to the displacement components $u^{(i)}$ through the relation

$$
\sigma_{j 3}^{(i)}=\mu_{i} \frac{\partial u^{(i)}}{\partial x_{j}}, i, j=1,2,
$$

with $\mu_{i}$ denoting the Lamé constants, and $\rho_{i}$ the mass densities of the layers. Since, only the antiplane motions are considered, the displacement vector employed above is taken as $\boldsymbol{u}=\left(0,0, u_{3}\left(x_{1}, x_{2}\right)\right) \equiv$ $u^{(i)}$ and hence there is no reference to the coordinate $x_{3}$ in the remaining of the paper. In what follows, the indices $i=1,2$ will correspond to the inner and outer layers, respectively. Along the interfaces of the layers we impose the continuity of stresses and displacements, i.e.

$$
\sigma_{23}^{(1)}=\sigma_{23}^{(2)} \quad \text { and } \quad u^{(1)}=u^{(2)} \text { at } x_{2}= \pm h_{1},
$$

together with the traction free outer faces given as

$$
\sigma_{23}^{(2)}=0 \quad \text { at } \quad x_{2}= \pm\left(h_{1}+h_{2}\right)
$$

It is a straightforward matter to reduce equation (1) to a couple of wave equations for the displacements given by (see, for example, [15])

$$
\Delta u^{(i)}-\frac{1}{c_{2 i}^{2}} \frac{\partial^{2} u^{(i)}}{\partial t^{2}}=0, \quad i=1,2
$$


with the wave velocities $c_{2 i}=\sqrt{\mu_{i} / \rho_{i}}, i=1,2$, corresponding, respectively, to inner and outer layers.

Assuming that the displacements have a time-harmonic dependence, the dispersion relation of the antisymmetric modes corresponding to the formulated problem turn out to be the same as equation (6) of [13], and it is produced here for ease of reference:

$$
\mu \alpha_{1} \cosh \left(\alpha_{1}\right) \cosh \left(\alpha_{2} h\right)+\alpha_{2} \sinh \left(\alpha_{1}\right) \sinh \left(\alpha_{2} h\right)=0
$$

where

$$
\alpha_{1}=\sqrt{K^{2}-\Omega^{2}}, \quad \alpha_{2}=\sqrt{K^{2}-\frac{\mu}{\rho} \Omega^{2}},
$$

and the dimensionless frequency $\Omega$ and the wavenumber $K$ are introduced through the transverse wave speeds and the thickness of the inner layer as

$$
\Omega=\frac{\omega h_{1}}{c_{21}}, \quad K=k h_{1} .
$$

with $\omega$ denoting the angular frequency and $k$ is the wavenumber. The remaining principal problem parameters are defined by the equations

$$
\mathrm{h}=\frac{h_{2}}{h_{1}}, \quad \mu=\frac{\mu_{1}}{\mu_{2}}, \quad \rho=\frac{\rho_{1}}{\rho_{2}} .
$$

The related stress and displacement components, on separating the time-harmonic factor $e^{i\left(k x_{1}-\omega t\right)}$, are found to be totally in agreement with equation (10) of [13] and are given by:

$$
\begin{aligned}
& u^{(1)}=h_{1} \frac{\sinh \left(\alpha_{1} \xi_{21}\right)}{\alpha_{1}}, \\
& \sigma_{13}^{(1)}=i \mu_{1} \frac{\sinh \left(\alpha_{1} \xi_{21}\right)}{\alpha_{1}}, \\
& \sigma_{23}^{(1)}=\mu_{1} \cosh \left(\alpha_{1} \xi_{21}\right)
\end{aligned}
$$

and

$$
\begin{aligned}
& u^{(2)}=h_{1} \beta\left(\cosh \left[\alpha_{2}\left(h \xi_{22}+1\right)\right]-\tanh \left[\alpha_{2}(h+1)\right] \sinh \left[\alpha_{2}\left(h \xi_{22}+1\right)\right]\right), \\
& \sigma_{13}^{(2)}=i \mu_{2} K \beta\left(\cosh \left[\alpha_{2}\left(h \xi_{22}+1\right)\right]-\tanh \left[\alpha_{2}(h+1)\right] \sinh \left[\alpha_{2}\left(h \xi_{22}+1\right)\right]\right), \\
& \sigma_{23}^{(2)}=\mu_{2} \alpha_{2} \beta\left(\sinh \left[\alpha_{2}\left(h \xi_{22}+1\right)\right]-\tanh \left[\alpha_{2}(h+1)\right] \cosh \left[\alpha_{2}\left(h \xi_{22}+1\right)\right]\right),
\end{aligned}
$$

where

$$
\beta=\frac{\sinh \alpha_{1}}{\alpha_{1}\left(\cosh \alpha_{2}-\sinh \alpha_{2} \tanh \left[\alpha_{2}(h+1)\right]\right)} .
$$

It should be remarked here that the parameters (9) are vital to the investigation of the dispersion equation (6) and it is only possible, on the basis of the choices of the orders of magnitudes of these parameters in advance, that a solution may be obtained. Below, we consider two layouts of contrasts, namely, stiff, thick outer layers with light, thin inner layer and soft, thin outer layers with stiff, thick inner layer. These configurations may be represented by the problem parameters (cf. eqn. (9)), as (see, also, [11]) 


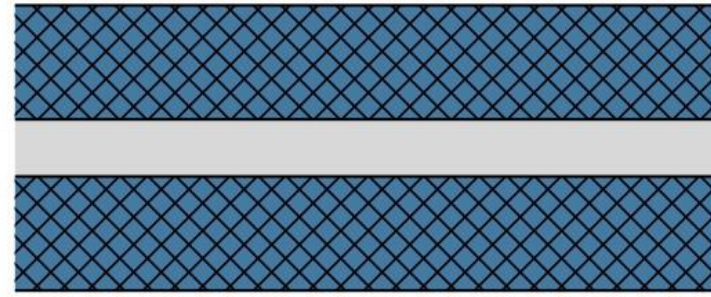

(a) $\mu \ll 1, h \sim \mu^{-\frac{1}{2}}, \rho \sim \mu^{\frac{1}{2}}$

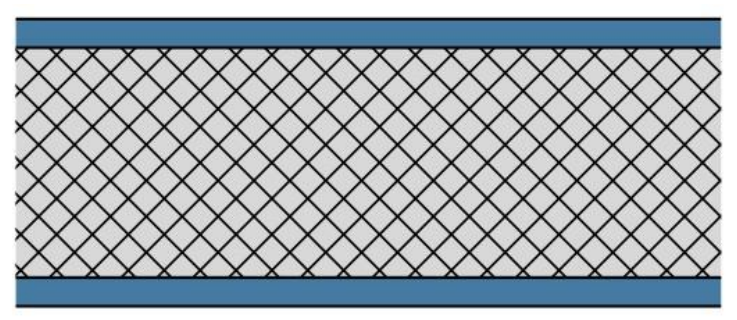

(b) $\mu \gg 1, h \sim \mu^{-2}, \rho \sim \mu^{-3}$

Figure 2. Configurations of different problem parameters: (a) stiff, thick outer layer with soft, thin inner layer; (b) soft, thin outer layers with stiff, thick inner layer.

\section{ASYMPTOTIC APPROACH}

Consider, first, setting $K=0$ in dispersion relation (6), resulting in the cut-off equation

$$
\tan (\Omega) \tan \left(h \sqrt{\frac{\mu}{\rho}}\right)=\sqrt{\mu \rho}
$$

which coincides with equation (15) of [13]. It follows from equation (13) that the low frequency range is

$$
\Omega\left(1+h \sqrt{\frac{\mu}{\rho}}\right) \ll 1
$$

predicting a single cut-off frequency given by

$$
\Omega \approx\left(\frac{\rho}{h}\right)^{1 / 2}
$$

provided that

$$
\rho \ll h \ll \mu^{-1} .
$$

Let us examine the long-wave motions for which

$$
K(1+h) \ll 1,
$$

over low-frequency range (14). Next, by expanding all trigonometric functions in (6) in Taylor series for small $\Omega$ and $K$ in the ranges given above, we derive a polynomial dispersion relation written as

$$
\mu+\gamma_{1} K^{2}+\gamma_{2} K^{4}+\gamma_{3} K^{2} \Omega^{2}+\gamma_{4} \Omega^{2}+\gamma_{5} \Omega^{4}+\cdots=0 .
$$

The coefficients of the polynomial expansion (18) are given by

$$
\begin{aligned}
& \gamma_{1}=\frac{\mu}{2}\left(1+h^{2}\right)+h, \\
& \gamma_{2}=\frac{\mu}{24}\left(1+6 h^{2}+h^{4}\right)+\frac{h}{6}\left(1+h^{2}\right),
\end{aligned}
$$




$$
\begin{aligned}
& \gamma_{3}=-\frac{\mu}{12}\left(1+3 h^{2}\right)-\frac{h}{6}-\frac{\mu h}{12 \rho}(2+3 \mu h)-\frac{\mu h^{3}}{12 \rho}(4+\mu h), \\
& \gamma_{4}=-\frac{\mu}{2}-\frac{\mu h}{\rho}\left(1+\frac{\mu h}{2}\right) \\
& \gamma_{5}=\frac{\mu}{24}+\frac{\mu h}{12 \rho}(2+3 \mu h)+\frac{\mu^{2} h^{3}}{24 \rho^{2}}(4+\mu h) .
\end{aligned}
$$

Dispersion curves for the exact dispersion relation (6) were plotted in Figs. 2 and 3 of [13] for contrast and non-contrast configurations and will be omitted here. Below, shortened forms of the polynomial dispersion relation will be obtained for configurations (a) and (b), i.e., for the different settings of the contrasts as well as the thicknesses of the layers and the obtained results will be compared with the exact dispersion curves which will be computed from equation (6).

\section{STIFF OUTER LAYERS WITH THIN LIGHT INNER LAYER}

For parameter setup (a) (see Figure 2), the coefficients of the polynomial dispersion relation (18) may be written, considering the problem parameters $\mu \ll 1, h \sim \mu^{-\frac{1}{2}}, \rho \sim \mu^{\frac{1}{2}}$, together with formulae (19), as

$$
\gamma_{1} \sim \mu^{-\frac{1}{2}}, \gamma_{2} \sim \mu^{-\frac{3}{2}}, \gamma_{3} \sim \mu^{-1}, \gamma_{4} \sim 1, \gamma_{5} \sim \mu^{-\frac{1}{2}}
$$

The leading order behaviour of each coefficient is thus given by

$$
\begin{aligned}
\gamma_{1} & =\frac{h_{\mu}}{\sqrt{\mu}}, \\
\gamma_{2} & =\mu^{-\frac{3}{2}} \frac{h_{\mu}}{6}, \\
\gamma_{3} & =-\frac{1}{3 \mu} \frac{h_{\mu}^{3}}{\rho_{\mu}}, \\
\gamma_{4} & =-\frac{h_{\mu}}{\rho_{\mu}}, \\
\gamma_{5} & =\frac{1}{\sqrt{\mu}} \frac{h_{\mu}^{3}}{\rho_{\mu}^{2}},
\end{aligned}
$$

where $h_{\mu}=h \sqrt{\mu} \sim 1$ and $\rho_{\mu}=\rho / \sqrt{\mu} \sim 1$. Consequently, equation (18) may conveniently be shortened as

$$
\mu+\frac{h_{\mu}}{\sqrt{\mu}} K^{2}-\frac{h_{\mu}}{\rho_{\mu}} \Omega^{2}=0
$$

Let us employ the scaling $\Omega^{2}=\mu^{\alpha} \Omega_{*}^{2}$ and $K^{2}=\mu^{\beta} K_{*}^{2}$, in equation (22), where the starred quantities are order of unity, i.e., $\Omega_{*} \sim K_{*} \sim 1$. Taking $\alpha=1$, and $\beta=3 / 2$ in the last equation, we obtain relation (22). On the other hand, when $0<\alpha<1$ and $\beta>\alpha+1 / 2$, these intervals of parameters cover the whole long-wave low-frequency band, which means that the shortened dispersion relation is satisfied in case $\Omega \ll 1$, and $K \ll 1$, see (14) and (16).

Rewriting dispersion relation (22) in terms of $\Omega_{*}$ and $K_{*}$, we arrive at 
Rewriting dispersion relation (22) in terms of $\Omega_{*}$ and $K_{*}$, we arrive at

$$
\Omega_{*}^{2}=\rho_{\mu}\left(\frac{\mu^{\frac{1}{2}-\alpha}}{h}+\mu^{\beta-\alpha-\frac{1}{2}} K_{*}^{2}\right)
$$

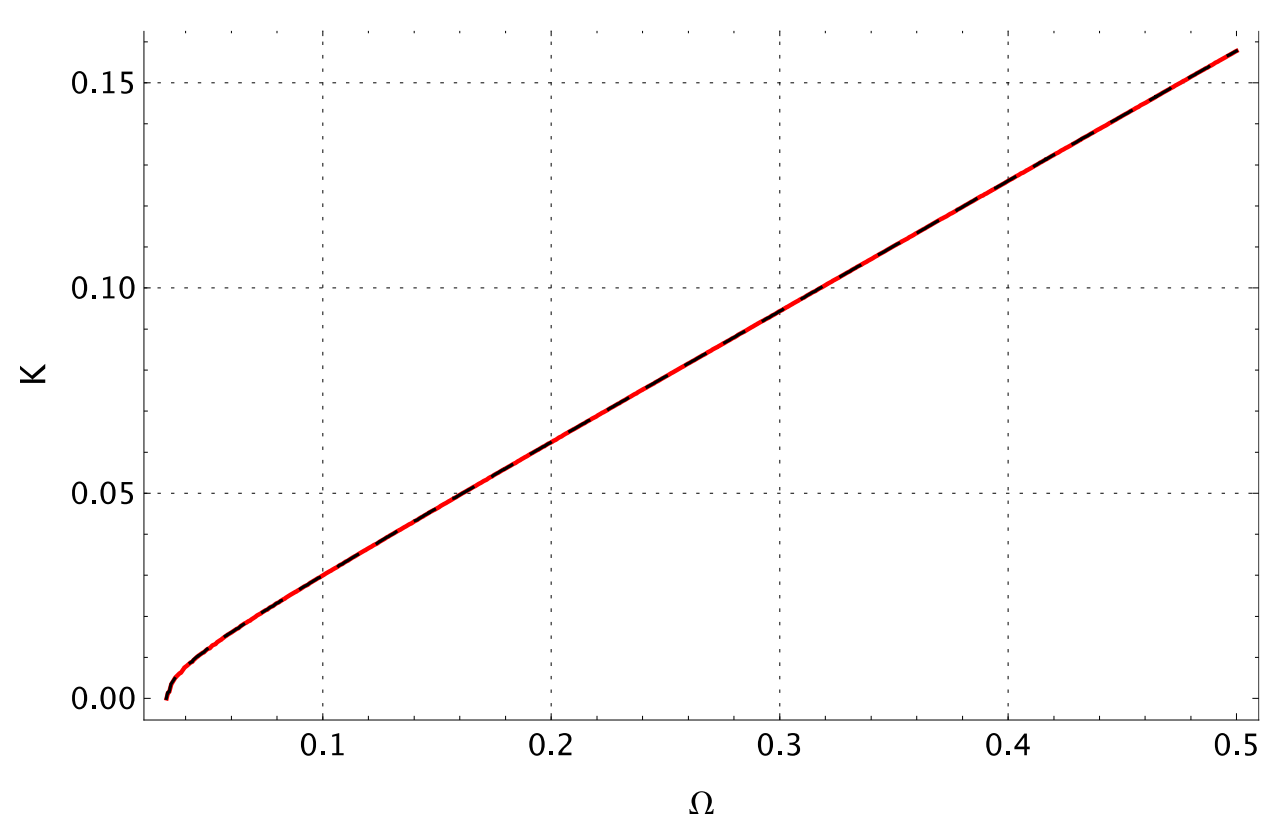

Figure 3. Comparison of the shortened polynomial equation (22) (red line) with the exact dispersion relation (6) (black dashed line) for the lowest antisymmetric vibration modes for configuration (a).

Figure 3 illustrates the comparison of the dispersion curve (22) with the original dispersion equation (6) for the set of parameters $\mu=0,001, \rho=0,01, h=10$. Even though the shortened dispersion relation is derived for a very short range of $\Omega$ and $K$, a remarkable agreement is observed over a wide frequency range.

\section{SOFT THIN OUTER LAYERS WITH LIGHT INNER LAYER}

In the case of configuration (b) (see, Figure 2), first, dividing the dispersion relation (18) by $\mu^{2}$ and setting $\eta=1 / \mu$, we find a set of new coefficients $\hat{\gamma}_{i}=\gamma_{i} / \mu^{2}$ as

$$
\hat{\gamma}_{1} \sim \eta, \hat{\gamma}_{2} \sim \eta, \hat{\gamma}_{3} \sim \hat{\gamma}_{4} \sim \hat{\gamma}_{5} \sim 1
$$

Taking into account the orders of the expansions of the coefficients $\hat{\gamma}_{j}, j \neq 4$, we realise that, in this case, a two-term expansion for the coefficient $\hat{\gamma}_{4}$ is required. Therefore, at leading order, the expansion of coefficients $\hat{\gamma}_{j}$ are represented as

$$
\begin{aligned}
& \hat{\gamma}_{1}=\frac{\eta}{2}, \\
& \hat{\gamma}_{2}=\frac{\eta}{24}, \\
& \hat{\gamma}_{3}=-\frac{1}{6} \frac{h_{\eta}}{\rho_{\eta}}, \\
& \hat{\gamma}_{4}=-\frac{h_{\eta}}{\rho_{\eta}}-\frac{\eta}{2}-\frac{\eta}{2} \frac{h_{\eta}^{2}}{\rho_{\eta}},
\end{aligned}
$$


Erbaş / Eskişehir Technical Univ. J. of Sci. and Tech. A - Appl. Sci. and Eng. 19 (4) - 2018

$$
\hat{\gamma}_{5}=\frac{1}{6} \frac{h_{\eta}}{\rho_{\eta}}+\frac{1}{6} \frac{h_{\eta}^{3}}{\rho_{\eta}^{2}}
$$

where $h_{\eta}=h / \eta^{2}$ and $\rho_{\eta}=\rho / \eta^{3}$. The dispersion relation (18) thus reduces to the polynomial equation

$$
\eta+\frac{\eta}{2} K^{2}-\frac{1}{6} \frac{h_{\eta}}{\rho_{\eta}} K^{2} \Omega^{2}-\left(\frac{h_{\eta}}{\rho_{\eta}}+\frac{\eta}{2}+\frac{\eta}{2} \frac{h_{\eta}^{2}}{\rho_{\eta}}\right) \Omega^{2}+\frac{1}{6}\left(\frac{h_{\eta}}{\rho_{\eta}}+\frac{h_{\eta}^{3}}{\rho_{\eta}^{2}}\right) \Omega^{4}=0 .
$$

We now normalize the wavenumber and the frequency by

$$
K^{2}=\eta K_{*}^{2} \quad \text { and } \quad \Omega^{2}=\eta \Omega_{*}^{2} .
$$

Inserting back into equation (26) we arrive at

$$
\eta+\frac{\eta^{2}}{2} K_{*}^{2}-\frac{\eta^{2}}{6} \frac{h_{\eta}}{\rho_{\eta}} K_{*}^{2} \Omega_{*}^{2}-\eta\left(\frac{h_{\eta}}{\rho_{\eta}}+\frac{\eta}{2}+\frac{\eta}{2} \frac{h_{\eta}^{2}}{\rho_{\eta}}\right) \Omega_{*}^{2}+\frac{\eta^{2}}{6}\left(\frac{h_{\eta}}{\rho_{\eta}}+\frac{h_{\eta}^{3}}{\rho_{\eta}^{2}}\right) \Omega_{*}^{4}=0
$$

and suggest a near cut-off asymptotic expansion given in the following form

$$
\Omega_{*}^{2}=\Omega_{0}^{2}+\eta \Omega_{1}^{2}+\cdots
$$

On substituting the latter into (28), we find

$$
\Omega_{0}^{2}=\rho_{\eta} / h_{\eta} \quad \text { and } \quad \Omega_{1}^{2}=\frac{\rho_{\eta}}{3 h_{\eta}}\left(K_{*}^{2}-h_{\eta}-\frac{\rho_{\eta}}{h_{\eta}}\right),
$$

from which follows the optimal shortened dispersion relation

$$
\Omega^{2}-\eta \frac{\rho_{\eta}}{3 h_{\eta}} K^{2}+\eta^{2} \frac{\rho_{\eta}}{3}+\frac{\eta^{2}}{3} \frac{\rho_{\eta}^{2}}{h_{\eta}^{2}}-\eta \frac{\rho_{\eta}}{h_{\eta}}=0
$$




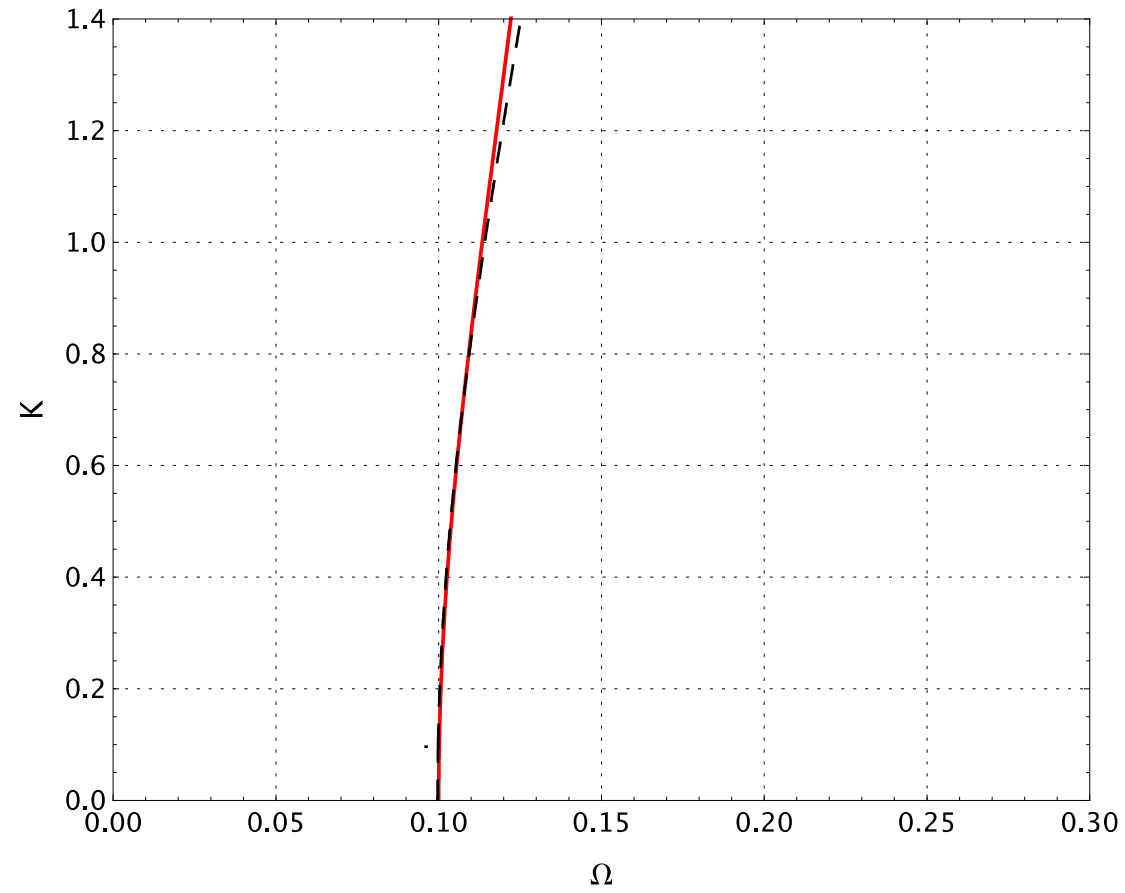

Figure 4. Comparison of the shortened polynomial equation (31) (red line) with the exact dispersion relation (6) (black dashed line) for the lowest antisymmetric vibration modes for configuration (a).

Figure 4 demonstrates that, contrary to configuration (a), the shortened dispersion equation (31) almost immediately approaches its upper bound of long-wave region bifurcating from the exact dispersion relation (6) for the problem parameters $\mu=100, \rho=0,000001$ and $h=0,0001$.

\section{ASYMPTOTIC DERIVATION OF THE EQUATIONS OF MOTION}

In this section, we carry out the derivation of the equations of motion (1) in their approximate forms. We will omit the configuration (a) as it completely agrees with the first setup of the paper (cf. eqn. (13) of [13]) and perform the analysis only for configuration (b). Let us first scale longitudinal coordinate and time by

$$
x_{1}=\frac{h_{1}}{\sqrt{\eta}} \xi_{1} \quad \text { and } \quad t=\frac{h_{1}}{\sqrt{\eta} c_{21}} \tau
$$

and use the scaling of the coordinate variables as

$$
\begin{aligned}
& \xi_{21}=\frac{x_{2}}{h_{1}}, 0 \leq x_{2} \leq h_{1}, \\
& \xi_{22}=\frac{x_{2}-h_{1}}{h_{2}}, \quad h_{1} \leq x_{2} \leq h_{1}+h_{2} .
\end{aligned}
$$

Also, we normalise displacements and stresses as

$$
u^{(i)}=h_{1} v^{(i)}, \quad \sigma_{13}^{(i)}=\mu_{i} \sqrt{\eta} S_{13}^{(i)}, \sigma_{23}^{(i)}=\mu_{1} S_{23}^{(i)}, \quad i=1,2 .
$$

Inserting equations (32)-(34) into the governing equations (1)-(4) in Section 2 we obtain, for the inner and outer layers, respectively 


$$
\begin{gathered}
\eta \frac{\partial S_{13}^{(1)}}{\partial \xi_{1}}+\frac{\partial S_{23}^{(1)}}{\partial \xi_{21}}+\eta \Omega_{0}^{2} v^{(1)}-\eta^{2} L\left(v^{(1)}\right)=0 \\
S_{13}^{(1)}=\frac{\partial v^{(1)}}{\partial \xi_{1}}, \quad S_{23}^{(1)}=\frac{\partial v^{(1)}}{\partial \xi_{21}}
\end{gathered}
$$

and

$$
\begin{gathered}
\eta \frac{\partial S_{13}^{(2)}}{\partial \xi_{1}}+\frac{1}{\eta^{3} h_{\eta}} \frac{\partial S_{23}^{(2)}}{\partial \xi_{22}}+\frac{1}{\eta^{3} \rho_{\eta}} \Omega_{0}^{2} v^{(2)}-\frac{1}{\eta^{2} \rho_{\eta}} L\left(v^{(2)}\right)=0 \\
S_{13}^{(2)}=\frac{\partial v^{(2)}}{\partial \xi_{1}}, \quad \eta h_{\eta} S_{23}^{(2)}=\frac{\partial v^{(2)}}{\partial \xi_{22}}
\end{gathered}
$$

where $\Omega_{0}$ is a frequency to be found and the operator $L$ is defined as

$$
L\left(v^{(i)}\right)=\frac{1}{\eta}\left(\Omega_{0} v^{(i)}+\frac{\partial^{2} v^{(i)}}{\partial \tau^{2}}\right), \quad i=1,2 .
$$

The continuity conditions at the interfaces of the layers together with the boundary conditions on the outer surfaces take the form

$$
\begin{aligned}
& \left.v^{(1)}\right|_{\xi_{21}=1}=\left.v^{(2)}\right|_{\xi_{22}=0}, \\
& \left.S_{23}^{(1)}\right|_{\xi_{21}=1}=\left.S_{23}^{(2)}\right|_{\xi_{22}=0},
\end{aligned}
$$

and

$$
\left.S_{23}^{(2)}\right|_{\xi_{22}=1}=0
$$

The displacements and stresses may be expanded in the asymptotic series as

$$
\begin{gathered}
v^{(i)}=v_{0}^{(i)}+\eta v_{1}^{(i)}+\cdots, \\
S_{j 3}^{(i)}=S_{j 3,0}^{(i)}+\eta S_{j 3,1}^{(i)}+\cdots, \quad i, j=1,2 .
\end{gathered}
$$

Inserting expansions (40) in formulae (36)-(39), we arrive, at leading order, at

$$
S_{13,0}^{(1)}=\frac{\partial v_{0}^{(1)}}{\partial \xi_{1}}, \quad S_{23,0}^{(1)}=\frac{\partial v_{0}^{(1)}}{\partial \xi_{21}}, \quad \frac{\partial S_{23,0}^{(1)}}{\partial \xi_{21}}=0
$$

and

$$
\begin{gathered}
\frac{1}{h_{\eta}} \frac{\partial S_{23,0}^{(2)}}{\partial \xi_{22}}+\frac{1}{\rho_{\eta}} \Omega_{0}^{2} v_{0}^{(2)}=0, \\
S_{13,0}^{(2)}=\frac{\partial v_{0}^{(2)}}{\partial \xi_{1}}, \quad \frac{\partial v_{0}^{(2)}}{\partial \xi_{22}}=0
\end{gathered}
$$

with the continuity conditions given by 


$$
\begin{gathered}
\left.v_{0}^{(1)}\right|_{\xi_{21}=1}=\left.v_{0}^{(2)}\right|_{\xi_{22}=0}, \\
\left.S_{23,0}^{(1)}\right|_{\xi_{21}=1}=\left.S_{23,0}^{(2)}\right|_{\xi_{22}=0},
\end{gathered}
$$

and the boundary condition as

$$
\left.S_{23,0}^{(2)}\right|_{\xi_{22}=1}=0
$$

We deduce from the last equation of (41) and (43), respectively,

$$
S_{23,0}^{(1)}=p_{1}\left(\xi_{1}, \tau\right) \text { and } v_{0}^{(2)}=w_{2}\left(\xi_{1}, \tau\right)
$$

and also notice that $\Omega_{0}$ agrees with the leading order term in the near cut-off expansion in (28). Consequently, we obtain

$$
S_{13,0}^{(1)}=\xi_{21} \frac{\partial w_{2}}{\partial \xi_{1}} \text { and } v_{0}^{(1)}=\xi_{21} p_{1}
$$

and

$$
S_{13,0}^{(2)}=\frac{\partial w_{2}}{\partial \xi_{1}} \text { and } S_{23,0}^{(2)}=w_{2}\left(1-\xi_{22}\right) \text {. }
$$

The near cut-off layout proposes to consider the next asymptotic order. We thus arrive at the equations

$$
\begin{gathered}
\frac{\partial S_{13,0}^{(1)}}{\partial \xi_{1}}+\frac{\partial S_{23,1}^{(1)}}{\partial \xi_{21}}+\Omega_{0}^{2} v_{0}^{(1)}=0 \\
S_{13,1}^{(1)}=\frac{\partial v_{1}^{(1)}}{\partial \xi_{1}}, \quad S_{23,1}^{(1)}=\frac{\partial v_{1}^{(1)}}{\partial \xi_{21}}
\end{gathered}
$$

and

$$
\begin{gathered}
\frac{1}{h_{\eta}} \frac{\partial S_{23,1}^{(2)}}{\partial \xi_{22}}+\frac{1}{\rho_{\eta}} \Omega_{0}^{2} v_{1}^{(2)}-\frac{1}{\rho_{\eta}} L\left(v_{0}^{(2)}\right)=0, \\
S_{13,1}^{(2)}=\frac{\partial v_{1}^{(2)}}{\partial \xi_{1}}, \quad S_{23,0}^{(2)}+\frac{1}{h_{\eta}} \frac{\partial v_{1}^{(2)}}{\partial \xi_{22}}=0
\end{gathered}
$$

with

$$
\begin{gathered}
\left.v_{1}^{(1)}\right|_{\xi_{21}=1}=\left.v_{1}^{(2)}\right|_{\xi_{22}=0}, \\
\left.S_{23,1}^{(1)}\right|_{\xi_{21}=1}=\left.S_{23,1}^{(2)}\right|_{\xi_{22}=0}
\end{gathered}
$$

and 


$$
\left.S_{23,1}^{(2)}\right|_{\xi_{22}=1}=0
$$

The obtained equations are then integrated through thickness and considering (47) and (48), it is established that $w_{2}=p_{1}$ and also

$$
\begin{aligned}
S_{13,1}^{(1)} & =-\frac{\xi_{21}^{3}}{6}\left(\frac{\partial^{3} w_{2}}{\partial \xi_{1}^{3}}+\frac{\rho_{\eta}}{h_{\eta}} \frac{\partial w_{2}}{\partial \xi_{1}}\right)+\xi_{21} \frac{\partial C_{2}\left(\xi_{1}, \tau\right)}{\partial \xi_{1}} \\
S_{23,1}^{(1)} & =-\frac{\xi_{21}^{2}}{2}\left(\frac{\partial^{2} w_{2}}{\partial \xi_{1}^{2}}+\frac{\rho_{\eta}}{h_{\eta}} w_{2}\right)+C_{2}\left(\xi_{1}, \tau\right) \\
v_{1}^{(1)} & =-\frac{\xi_{21}^{3}}{6}\left(\frac{\partial^{2} w_{2}}{\partial \xi_{1}^{2}}+\frac{\rho_{\eta}}{h_{\eta}} w_{2}\right)+\xi_{21} C_{2}\left(\xi_{1}, \tau\right) \\
S_{13,1}^{(2)} & =h_{\eta} \frac{\partial w_{2}}{\partial \xi_{1}}\left(\xi_{22}-\frac{\xi_{22}^{2}}{2}\right)+\frac{\partial D_{1}\left(\xi_{1}, \tau\right)}{\partial \xi_{1}} \\
S_{23,1}^{(2)} & =\frac{h_{\eta}}{\rho_{\eta}} L\left(w_{2}\right)\left(\xi_{22}-1\right)-\frac{h_{\eta}}{6} w_{2}\left(1-\xi_{22}^{3}\right)+\frac{h_{\eta}}{2} w_{2}\left(1-\xi_{22}^{2}\right)+D_{1}\left(\xi_{1}, \tau\right)\left(1-\xi_{22}\right), \\
v_{1}^{(2)} & =h_{\eta} w_{2}\left(\xi_{22}-\frac{\xi_{22}^{2}}{2}\right)+D_{1}\left(\xi_{1}, \tau\right)
\end{aligned}
$$

with the constant $C_{2}$ given by

$$
C_{2}\left(\xi_{1}, \tau\right)=\frac{1}{6}\left(\frac{\partial^{2} w_{2}}{\partial \xi_{1}^{2}}+\frac{\rho_{\eta}}{h_{\eta}} w_{2}\right)+D_{1}\left(\xi_{1}, \tau\right)
$$

and the sought for function $w_{2}$ satisfies the one-dimensional equation

$$
\frac{1}{3} \frac{\partial^{2} w_{2}}{\partial \xi_{1}^{2}}+w_{2}\left(\frac{h_{\eta}}{3}+\frac{1}{3} \frac{h_{\eta}}{\rho_{\eta}}\right)-\frac{h_{\eta}}{\rho_{\eta}} L\left(w_{2}\right)=0
$$

The latter equation may easily be written in terms of the original variables and takes the form

$$
\frac{1}{3} h_{1}^{2} \frac{\mu_{1}}{\mu_{2}} \frac{\partial^{2} u^{2}}{\partial x_{1}^{2}}-\frac{h_{1} h_{2} \rho_{2}}{\mu_{2}} \frac{\partial^{2} u^{2}}{\partial t^{2}}+\left(\frac{1}{3} \frac{h_{2} \mu_{1}^{2}}{h_{1} \mu_{2}^{2}}+\frac{1}{3} \frac{h_{2} \mu_{2} \rho_{1}}{h_{1} \mu_{1} \rho_{2}}-\frac{\mu_{1}}{\mu_{2}}\right) u^{2}=0 .
$$

The associated dispersion relation may be presented in the original variables as

$$
\frac{1}{3} h_{1}^{2} \frac{\mu_{1}}{\mu_{2}} k^{2}-\frac{h_{1} h_{2} \rho_{2}}{\mu_{2}} \omega^{2}-\left(\frac{1}{3} \frac{h_{2} \mu_{1}^{2}}{h_{1} \mu_{2}^{2}}+\frac{1}{3} \frac{h_{2} \mu_{2} \rho_{1}}{h_{1} \mu_{1} \rho_{2}}-\frac{\mu_{1}}{\mu_{2}}\right)=0 .
$$

This, as might be expected, agrees with the shortened dispersion relation (31) obtained in Section 5.

\section{CONCLUSION}

Two asymptotic formulas governing the antiplane deformation of a three-layered plate of infinite extent corresponding to two different configurations of contrast problem parameters are constructed. It 
is shown that the first of these is valid over the whole low-frequency range, whereas the second configuration is valid only in a close neighbourhood of the lowest cut-off frequency. For both cases, the exact dispersion relation (6) is reduced to polynomial dispersion relations given by (22) and (26) which, for the second configuration, is further reduced to the so-called shortened dispersion relation (31). The accuracy of the approximate equations is numerically compared to the transcendental equation (6) and they are depicted in Figures 3 and 4 demonstrating the validity of the obtained results. The asymptotic equations of motion are derived only for the second configuration since, in the first case, the obtained equation agrees fully with the asymptotic formula presented in [13]. It should also be remarked that the dispersion relations obtained from the asymptotic equations of motion coincides with the shortened dispersion relations.

The approach employed in the paper may be extended to multi-layered structures as well as asymmetric geometries and 3D problems. One particular problem of interest is the investigation of St. Venant principle for a layered semi-infinite elastic structure for which an extra boundary condition will also result in additional parameters to be taken into account. The asymptotic analysis of a layered semi-infinite elastic structure will generalise the classical the St. Venant principle for an elastic strip of semi-infinite extent.

\section{REFERENCES}

[1] Vinson JR. The Behaviour of Sandwich Structure of Isotropic and Composite Materials, CRC Press, 1999.

[2] Altenbach H, Eremeyev VA, Naumenko K. On the use of the first order shear deformation plate theory for the analysis of three-layer plates with thin soft core layer. ZAMM-J.Appl. Math. Mech./Zeitsch. Ange. Mathe. Mech. 2015, 95.10: 1004-1011.

[3] Naumenko K, Eremeyev VA. A layer-wise theory for laminated glass and photovoltaic panels. Composite Structures 2014, 112: 283-291.

[4] Martin TP, Layman CN, Moore KM and Orris GJ. Elastic shells with high-contrast material properties as acoustic metamaterial components. Physical Review B 2012, 85.16: 161103.

[5] Lee P, Chang N. Harmonic waves in elastic sandwich plates. Journal of Elasticity 1979, 9.1: 5169.

[6] Wang CM, Reddy JN and Lee KH. Shear deformable beams and plates: Relationships with classical solutions, Elsevier, 2000.

[7] Milton GW. The theory of composites, Cambridge University Press, 2002.

[8] Hohe J, Librescu L. Advances in the structural modelling of elastic sandwich panels. Mechanics of Advanced Materials and Structures 2004, 11.4-5: 395-424.

[9] Berdichevsky VL. An asymptotic theory of sandwich plates. International Journal of Engineering Science 2010, 48.3: 383-404.

[10] Kaplunov J, Prikazchikov DA and Sergushova O. Multi-parametric analysis of the lowest natural frequencies of strongly inhomogeneous elastic rods. Journal of Sound and Vibration 2016, 366: 264-276. 
[11] Kaplunov J, Prikazchikov DA and Prikazchikov LA. Dispersion of elastic waves in a strongly inhomogenous three-layered plate. International Journal of Solids and Structures 2017, 113:169179.

[12] Kaplunov J, Prikazchikov DA and Prikazchikov LA. Dispersion of elastic waves in laminated glass. Procedia Engineering 2017, 199:1489-1494.

[13] Prikazchikov LA, Aydın YE, Erbaş B and Kaplunov J. Asymptotic analysis of anti-plane dynamic problem for a three-layered strongly inhomogeneous laminate. Mathematics and Mechanics of Solids 2018, doi: 10.1177/1081286518790804.

[14] Ewing WM, Jardetzky WS. Elastic waves in layered media. 1st Ed. McGraw-Hill, 1957.

[15] Achenbach J. Wave propagation in elastic solids. Elsevier, 2012. 